\title{
Assessment of Beating Parameters in Human Induced Pluripotent Stem Cells Enables Quantitative In Vitro Screening for Cardiotoxicity
}

\author{
Oksana Sirenko ${ }^{1,{ }^{*}}$, Evan F. Cromwell ${ }^{1,{ }^{*}}$, Carole Crittenden ${ }^{1}$, Jessica A. Wignall ${ }^{2}$, Fred A. \\ Wright $^{3}$, and Ivan Rusyn ${ }^{2}$ \\ ${ }^{1}$ Molecular Devices LLC, Sunnyvale, CA 94089 \\ ${ }^{2}$ Department of Environmental Sciences and Engineering, University of North Carolina, Chapel \\ Hill, NC 27599 \\ ${ }^{3}$ Department of Biostatistics, University of North Carolina, Chapel Hill, NC 27599
}

\section{Abstract}

\begin{abstract}
Human induced pluripotent stem cell (iPSC)-derived cardiomyocytes show promise for screening during early drug development. Here, we tested a hypothesis that in vitro assessment of multiple cardiomyocyte physiological parameters enables predictive and mechanistically-interpretable evaluation of cardiotoxicity in a high-throughput format. Human iPSC-derived cardiomyocytes were exposed for 30 minutes or 24 hours to 131 drugs, positive (107) and negative (24) for in vivo cardiotoxicity, in up to 6 concentrations $(3 \mathrm{nM}$ to $30 \mu \mathrm{M})$ in 384-well plates. Fast kinetic imaging was used to monitor changes in cardiomyocyte function using intracellular $\mathrm{Ca}^{2+}$ flux readouts synchronous with beating, and cell viability. A number of physiological parameters of cardiomyocyte beating, such as beat rate, peak shape (amplitude, width, raise, decay, etc.) and regularity were collected using automated data analysis. Concentration-response profiles were evaluated using logistic modeling to derive a benchmark concentration (BMC) point-of-departure value, based on one standard deviation departure from the estimated baseline in vehicle $(0.3 \%$ dimethylsulfoxide)-treated cells. BMC values were used for cardiotoxicity classification and ranking of compounds. Beat rate and several peak shape parameters were found to be good predictors, while cell viability had poor classification accuracy. In addition, we applied the Toxicological Prioritization Index approach to integrate and display data across many collected parameters, to derive "cardiosafety" ranking of tested compounds. Multi-parameter screening of beating profiles allows for cardiotoxicity risk assessment and identification of specific patterns defining mechanism-specific effects. The data and analysis methods may be used widely for compound screening and early safety evaluation in the drug development process.
\end{abstract}

\footnotetext{
(C) 2013 Elsevier Inc. All rights reserved.

Corresponding authors: Oksana Sirenko ( oksana.sirenko@ moldev.com) or Evan F Cromwell ( evan.cromwell@ moldev.com), Molecular Devices LLC, 1311 Orleans Drive, Sunnyvale, CA 94089, USA.

Conflict of Interest:

O.S., E.F.C., and C.C. were employed by Molecular Devices, LLC, a company whose products and software were used to collect and analyze the data presented in this manuscript. J.A.W., F.A.W., and I.R. declare no conflicts of interest.

Publisher's Disclaimer: This is a PDF file of an unedited manuscript that has been accepted for publication. As a service to our customers we are providing this early version of the manuscript. The manuscript will undergo copyediting, typesetting, and review of the resulting proof before it is published in its final citable form. Please note that during the production process errors may be discovered which could affect the content, and all legal disclaimers that apply to the journal pertain.
} 


\section{Keywords}

Cardiotoxicity; iPSC cardiomyocytes; predictive toxicology; stem cell; calcium transient; ion channel; hERG; fast kinetic fluorescence imaging; screening

\section{Introduction}

Cardiotoxicity is a well-known side effect of many drugs and a leading cause of safetybased compound attrition in preclinical drug development (Kola and Landis, 2004; Laverty et al., 2011). Sensitive in vitro assays that can provide early detection of cardiovascular side effects have been the focus of toxicology research for several decades (Lawrence et al., 2008). Specifically, new cell models that better represent the in vivo environment, as well as new phenotypic readouts that are related to myocardial performance, are needed to develop a sensitive, specific, and predictive assay. Equally important is a requirement for assays to be amenable to high-throughput screening in a self-regenerating cell-based model. Thus, while primary cardiomyocytes or myocardial tissue preparations have been used, stem cellderived cardiomyocytes have become a popular model (Anson et al., 2011; Mercola et al., 2013; Scott et al., 2013). Human induced pluripotent stem cell (iPSC)-derived cardiomyocytes, an infinitely expandable source of cells from multiple individuals, are especially attractive, as they recapitulate the expected genetic, genomic, biochemical, mechanical, and electrophysiological behaviors of human cardiomyocytes and heart tissue (Guo et al., 2011; Cerignoli et al., 2012; Rana et al., 2012; Puppala et al., 2013; Sirenko et al., 2013).

Synchronous spontaneous rhythmic beating is a phenotype employed by most in vitro approaches for evaluating the cardiac-specific function. A wide array of methods is utilized in iPSC-based screening [reviewed in (Scott et al., 2013)], such as automated patch clamp, microelectrode arrays, mechanical movement, $\mathrm{Ca}^{2+}$ transients, and cellular impedence. Although the diversity of the detection methods creates an opportunity to screen specific phases of the contractile cascade, scalability of these assays to the high-throughput setting has not been addressed, as typically several dozen or fewer compounds are considered, and only over a limited range of concentration. One example of a successful integration of in vitro assays into a cardiotoxicity testing pipeline is the human Ether-a-go-go Related-Gene (hERG) potassium channel, a well-established molecular target for arrhythmia-causing drugs. High-throughput screening using recombinant hERG ion channels and radiolabelled ligands, combined with structure-activity modeling for hERG selectivity of drug candidates, is now used routinely in drug safety testing (Taboureau and Jorgensen, 2011).

Our previous study showed that iPSC-derived cardiomyocytes are amenable to automated assays for measuring the impact of drugs on the beating rate, mechanical activity, and intracellular calcium handling (Sirenko et al., 2013). By utilizing fast kinetic fluorescence imaging of intracellular $\mathrm{Ca}^{2+}$ levels, together with automated signal analysis software, we demonstrated that the phenotypic response of spontaneously contracting cardiomyocytes may be characterized with a variety of parameters indicative of the cardiac physiology. We reasoned that this assay, coupled with robotic liquid handling, is well-suited for quantitative high-throughput screening (HTS) and demonstrate the potential of this approach for investigative toxicology applications. Specifically, we tested a hypothesis that in vitro assessment of multiple cardiomyocyte physiological beating parameters enables predictive and mechanistically-interpretable evaluation of cardiotoxicity in a high-throughput format. We used a library of 131 compounds representing different groups of cardiotoxic and cardiosafe drugs to enable characterization of the classification accuracy of the assay. In 
addition we have proposed and validated methods for statistical analysis and ranking of compounds for cardiac safety.

\section{Methods}

Chemicals

A library of pharmacologically active compounds (Screen-Well Cardiotoxicity Library, BML-2850, ENZO Life Sciences, Farmingdale, NY) was used in these studies. The library (see Supplemental Table 1 for the list) contains 131 compounds representing blockers of $\mathrm{Na}^{+}, \mathrm{K}^{+}$and $\mathrm{Ca}^{2+}$ channels, hERG blockers, adreno receptor agonists and antagonists, dopamine receptor antagonists, histamine receptor antagonists, anti-inflammatory and antiviral drugs with known cardiac side effects. The library also includes a number of anticancer drugs, representing anthracyclines, kinase inhibitors, and receptor antagonists. In addition, there were 24 chemicals classified as non-toxic by the manufacturer. All compounds were prepared as $10 \mathrm{mM}$ stock in $100 \%$ dimethyl sulfoxide (DMSO).

\section{Human iPSC-derived cardiomyocytes and cell culture}

Human iPSC-derived cardiomyocytes (iCell ${ }^{\mathrm{TM}}$ Cardiomyocytes), plating medium, and maintenance medium were obtained from Cellular Dynamics International (Madison, WI). Differentiation protocols and cell culture conditions were as detailed previously in Ma $e t$ al. (Ma et al., 2011) and Sirenko et al. (Sirenko et al., 2013) Cells were plated into gelatin coated wells at 8,000 cells/well in 384 multi-well plates. Strong synchronous contractions of cells were evident after 7 days of culture. Experiments were conducted 8-10 days postplating on plates where all wells demonstrated regular synchronous contractions [on average 25 beats/minute, see Figure 2 and (Sirenko et al., 2013)]. Compound plates were prewarmed to $37^{\circ} \mathrm{C}$ before chemicals were added to cells. Concentration of DMSO was $0.1 \%$ in chemical and vehicle-only containing wells (except for $30 \mu \mathrm{M}$ wells where it was $0.3 \%$ ). In 30 min exposure experiments, FLIPR ${ }^{\circledR}$ Calcium Assay Kit 5 (Molecular Devices) reagents were added to each well and cells incubated for 30 minutes. Then, chemicals (in duplicates) or DMSO alone (30-38 wells/plate) were added to wells and cells were incubated for additional $30 \mathrm{~min}$. For $24 \mathrm{hr}$ exposure experiments, cells were incubated with chemicals for 23.5 hrs, FLIPR $®$ Calcium Assay Kit 5 or cell viability (see below) reagents added, and data acquired 30 min thereafter. All experiments were performed at $37^{\circ} \mathrm{C}$ and $5 \% \mathrm{CO}_{2}$. Data acquisition on FLIPR system was performed at $37^{\circ} \mathrm{C}$.

Imaging was used in parallel to assess cell viability. To determine viability, cells were incubated for 30 min with a mixture of three dyes: $2 \mu \mathrm{M}$ Calcein AM, $2 \mu \mathrm{g} / \mathrm{ml}$ Hoechst 33258, and $200 \mathrm{nM}$ of MitoTracker Orange (all from Invitrogen, Carlsbad CA). Images were acquired using ImageXpress ${ }^{\circledR}$ Micro XL automated microscope (Molecular Devices, Sunnyvale, $\mathrm{CA}$ ) using a $10 \mathrm{x}$ objective $(\mathrm{NA}=0.3)$. The light source was a solid state white light engine with emission from $380 \mathrm{~nm}$ to $680 \mathrm{~nm}$. A FITC filter cube (Ex 482/35, Em 536/40) (center wavelength(nm)/bandpass width(nm)) was used for Calcein AM. A TRITC filter cube (Ex. 543/22, Em 593/40) was used for MitoTracker Orange. A DAPI filter cube (Ex 377/50, Em 447/60) was used for Hoechst nuclear stain. Output parameters included total cell number, number of viable cells, and number of cells with intact mitochondria. As initial analysis showed high correlation between cell viability and mitochondria integrity (data not shown), we used only cell viability scoring (number of viable, i.e. Calcein AMpositive cells in each well) for further analysis.

\section{Data collection}

The FLIPR ${ }^{\circledR}$ Tetra high-throughput cellular screening system combined with a TetraCycler ${ }^{\mathrm{TM}}$ Microplate Handler (Molecular Devices) was used in these experiments. 
Fluorescence of FLIPR Calcium 5 dye (Ex. 485nm, Em. 530nm) was acquired at 8 frames per second (fps), and up to 800 image frames were collected while cells were maintained at $37^{\circ} \mathrm{C}$. The total time for image acquisition and analysis was approximately 2 min per plate. An automated algorithm in ScreenWorks ${ }^{\circledR}$ Peak Pro ${ }^{\mathrm{TM}}$ software (Molecular Devices) was used to process the data. Description of peak detection and fitting of a binomial function to calculate multivariate parameters are included in Sirenko et al. (Sirenko et al., 2013) Briefly, beat rate, peak amplitude, peak width (at 10\% amplitude), peak spacing, peak rise (time from $10 \%$ to $90 \%$ ), peak decay (time from $90 \%$ to $10 \%$ ), and peak irregularity were derived. Cell viability was assessed as a number of viable (Calcein AM positive) cells per field. Raw data, as well as data normalized to plate-specific vehicle (DMSO)-containing wells, for each endpoint are available as Supplemental Tables 2 and 3.

\section{Concentration-response curve fitting, derivation of benchmark concentrations and ranking of compounds}

Normalized data for each chemical and endpoint were used to fit a concentration-response quantitative logistic function and to visualize the concentration-response relationship, using the $R$ software version 2.15.2 (see Supplemental Methods for script). Variability of the DMSO-only wells was used to derive the benchmark concentration (BMC) (EPA, 2012), which involves modeling to obtain dose levels corresponding to specific response levels near the low end of the observable range of the data. An accepted approach for continuous data is to define a point-of-departure (i.e., benchmark) response as a change in the mean equal to one control standard deviation from the control mean, and thus we tested various cut-offs from 0.25 to 3 standard deviations at 0.25 intervals.

For comparing receiver operating characteristic (ROC curves) of classification accuracy of BMCs obtained at different points-of-departure we used visualization and partial area under the curve (AUC) calculations with the pROC R package (Robin et al., 2011). Receiver operating characteristic plots were created to illustrate the performance of a binary classifier (cardiotoxic vs. safe) by plotting the fraction of true positives out of the positives vs. the fraction of false positives out of the negatives at various threshold settings. The area under the curve of the ROC plot is then used as common metric to assess the classification accuracy of a classifier (Fawcett, 2006). The AUC derived from the ROC curves are equal to the probability that a classifier will rank a randomly chosen positive instance higher than a randomly chosen negative one.

An interactive Toxicological Prioritization Index $\left(\mathrm{ToxPi}^{\mathrm{TM}}\right.$ ) application (Reif et al., 2013) was used to integrate evidence across endpoints and to visualize the relative prioritized ranks of compounds.

\section{Chemical descriptors}

Two-dimensional chemical descriptors were calculated with Dragon software (v.5.5, Talete SRL, Milan, Italy). Descriptors were not calculated for two inorganic compounds (arsenic trioxide, cisplatin [cis-diamineplatinum(II)dichloride]) and two organometallic compounds (bortezomib, sodium stibogluconate). After removing descriptors with low variance $\left(\mathrm{SD}<10^{-6}\right)$ and highly correlated descriptors (if pairwise $r^{2}>1.0$, one of the pair was randomly removed), descriptors were range-scaled to the $[0,1]$ interval.

\section{Results}

\section{High-throughput cardiomyocyte beating assay}

We developed methods for measuring the impact of drug candidates on the beating rate of human iPSC derived cardiomyocytes using fast kinetic fluorescence imaging. 
Cardiomyocyte contraction rate and pattern are characterized by monitoring changes in intracellular $\mathrm{Ca}^{2+}$ measured using calcium sensitive dyes. The assay allows characterization of beating profiles by using multi-parameter analysis outputs such as beating rate, peak frequency and width, or waveform irregularities. (Sirenko et al., 2013). One of the major objectives of this study was to determine the feasibility of conducting the cardiomyocyte beating assay that employs fluorescent dyes to monitor changes in $\mathrm{Ca}^{2+}$ transients in a highthroughput format. Intracellular calcium ion fluxes are an integral part of the cardiomyocyte contraction process (Bers, 2002). Because $\mathrm{Ca}^{2+}$ transients are synchronous with cell beating (Qian and Guo, 2010; Sirenko et al., 2013), simultaneous monitoring of the beat rate, amplitude, and a multitude of other parameters affords evaluation of the physiological function of the heart (i.e., contractility) in vitro. Concordance between mechanical contraction and modulation of $\mathrm{Ca}^{2+}$ signal was demonstrated previously (Sirenko et al., 2013). The cells were cultured in a near-confluent monolayer and the average fluorescence intensity of all cells in a well was recorded over time. Typical images of cells going through a contraction cycle were observed [Supplemental Figure 1, see Sirenko et al. (Sirenko et al., 2013)]. The assay was optimized for a 384-well format to make it suitable for screening compound libraries. The assay variability was assessed for different output parameters on an inter- and intra-plate basis. Well-to-well coefficients of variation were $15 \%$ or less for most parameters, with the exception of peak amplitude. Visualization of the effects of chemicals on individual beating pattern parameters is shown in Figure 1. Overall concentrationresponse and compound similarities are evident from the heat map and hierarchical clustering for most of the compounds from the screened library identified as cardiotoxic (Figure 1, top), while little effect was observed with non-toxic compounds (Figure 1, bottom). When all screened compounds were combined and hierarchical clustering was performed, non-toxic compounds clustered (not shown) with some of the cardiotoxic agents that were without effect in this experiment (see below for a more detailed analysis of the classification accuracy and concentration-response analysis).

Even based on a very general clustering analysis we observed that known classes of cardiotoxic compounds had expected effects. For example, cardiac glycosides (e.g., digoxin and digitoxin) blocked beating completely at $3 \mu \mathrm{M}$. Antiarrythmic compounds $\left(\mathrm{Na}^{+}\right.$channel blockers lidocaine, disopyramide, flecainid acetate, and quinidine) had little effect on the beating rate, but affected spacing between peaks and regularity. Non-selective and selective $\alpha$ - and $\beta$-adrenergic receptor blockers (amiodarone, soltalol, pindolol) had negative chronotrope effect, while isoproterenol ( $\beta$-agonist) had positive chronotrope effect. $\mathrm{Ca}^{2+}$ channel blockers (bepridil, verapamil, diltiazem, and nicardipine) and anti-histamine compounds with known hERG blocker hazard (astemizol and terfenadine) strongly inhibited beat rate. At the same time, it should be noted that some compounds had hormesis-like concentration effects: beating rate increased at low concentrations, but decreased or completely stopped at high doses (e.g., digoxin, salmeterol). This pattern is consistent with positive chronotrope effects at low concentrations and toxic effects at higher concentrations.

A number of cardiotoxic compounds in this library did not have an effect at 30 minutes. Included in this group were several anti-inflammatory drugs (ibuprofen, meloxicam, indomethacin, diclofenac and rofecoxib), anti-diabetic drugs (rosiglitazone, pioglitazone) and most anti-cancer drugs that inhibit cell proliferation or cause apoptosis (doxorubicin, daunorubicin, cisplatin, 5-fluoracil).

As it is expected that different drugs may vary in the timing of their effects on cardiomyocytes (e.g., channel blockers/receptor antagonists $v s$. anti-cancer drugs), we used two time-points, $30 \mathrm{~min}$ and $24 \mathrm{hrs}$, to account for both types of toxicity. Separate plates were used for each time point. Importantly, beating rate and other parameters exhibited considerable concordance among $30 \mathrm{~min}$ and $24 \mathrm{hrs}$ time-points, both without treatment and 
upon exposure to compounds (Figure 1). Most compounds exhibited adverse effects at the same compound concentrations at either $30 \mathrm{~min}$, or $24 \mathrm{hrs}$. It should be noted, however, that cardiomyocyte beating is sensitive to media temperature and that these experiments should be performed under tight environmental control (data not shown).

\section{Analysis of the concentration response}

We tested the library of compounds in a 384-well plate format, in duplicates, using 6 and 4 concentrations for $30 \mathrm{~min}$ and $24 \mathrm{hrs}$ exposure, respectively (see Figure 1, or Supplemental Tables 2 and 3). We also found that up to $0.3 \%$ DMSO does not have an effect on the assessed parameters as compared to media-only wells (data not shown). Thus, we used 3038 wells/plate to estimate the variance in negative control data (i.e., vehicle-only treated cells).

The effective concentration at $50 \%$ of the maximum response $\left(\mathrm{EC}_{50}\right)$ is the reference point that is used most frequently in the analysis of in vitro data; however, the use of the $\mathrm{EC}_{50}$ may be problematic in cases when $50 \%$ response is far outside the range of concentrations tested. In addition, it is expected that not every tested chemical will elicit the "maximum effect" (for example a non-toxic compound), which complicates the process of fitting the Hill function or the equivalent parameter in other sigmoidal models. Because the vehicleonly effect level was normalized across plates, we performed four-parameter maximumlikelihood logistic modeling to fit the experimental data at test concentrations using duplicate measurements (see Figure 2 for a representative concentration-response analysis). In accordance with the US EPA guidance for dose-response modeling (EPA, 2012) and determination of the point-of-departure value, we selected a one standard deviation departure from the control mean as the benchmark response from which a BMC was derived (see Figure 2, top right panel). If the maximum response did not reach this level in the concentration range tested, the "no observable adverse effect level" was recorded as $30 \mu \mathrm{M}$, the highest concentration used. Identical curve fitting and BMC derivation procedures were applied to all chemicals and phenotypes collected (except for peak irregularity which is a binary parameter). For example, while disopyramide had no effect on the beat rate at the 30 min exposure time-point, it had a clear concentration-dependent effect on the peak shape parameters (Figure 2). No effect on cell viability, evaluated $24 \mathrm{hrs}$ after treatment, was observed for disopyramide.

\section{Classification of cardiotoxicity based on multiparametric phenotyping using iPSC-derived cardiomyocytes}

While the shape and frequency of $\mathrm{Ca}^{2+}$ transient peaks, as well as the information on cell viability, provide an opportunity to evaluate specific mechanisms of cardiotoxicity, a more global ranking of the potential hazard is also of potential value for decision-making in drug and chemical safety evaluation. First, we compared the effects of cardiotoxic vs non-toxic chemicals for each phenotype (Figure 3). BMCs for the beat rate and peak shape parameters were found to be significantly different between groups, while there was no difference in cell viability.

Second, we aimed to determine the classification accuracy (cardiotoxic vs non-toxic) of the BMC values in each parameter (Figure 4). We found that the highest classification accuracy, for the compound library that was tested, was achieved with the beat rate phenotype, with the receiver operating characteristic plot of sensitivity vs. specificity AUC of 0.8 and 0.91 for $30 \mathrm{~min}$ and $24 \mathrm{hrs}$ time-points, respectively. Cell viability and peak rise time were poor classifiers. To evaluate whether an alternate choice for the point-of-departure cutoff would affect classification accuracy, we derived BMCs at different fractions of standard deviation of the mean for vehicle-treated cells, from 0.25 to 3 in 0.25 increments. This analysis 
showed that a choice of one standard deviation generates consistently high classification accuracy among all phenotypes (Supplemental Table 4).

Third, because strong structure-activity relationships have been established between inherent chemical properties and certain types of cardiotoxicity (Frid and Matthews, 2010), we conducted principal component analysis of cardiotoxic $v s$. non-toxic compounds in the tested library based on the chemical descriptors (Figure 5A). From the plot of the first two principal components it is evident that non-toxic compounds were largely representative of the chemical space of the entire chemical library. In addition, we used principal component analysis to visualize the distribution of cardiotoxic and non-toxic compounds using multiparametric assessment of iPSC-derived cardiomyocyte beating patterns (Figures 5B and C). Clearly, non-toxic compounds showed response profiles that were more similar to each other than to most cardiotoxic compounds at either time point. Most of the signal in principal component 1 for the 30 min time-point (Figure 5B) was contributed by drugs that had dopamine- and/or histamine-ergic properties (triangles); while principal component 2 was largely separating ion $\left(\mathrm{Na}^{+}, \mathrm{K}^{+}\right.$, or $\left.\mathrm{Ca}^{2+}\right)$ channel blockers from other compounds (diamonds).

\section{Ranking of compounds for cardiotoxicity potential}

We have shown previously that significant correlation exists between the effect levels derived from the in vitro analysis of cardiac beating parameters and both Cmax and results of other assays for cardiotoxicity (Sirenko et al., 2013). While comparisons of in vitroderived effect levels to Cmax has been suggested as a surrogate metric for ranking compounds with respect to their potential in vivo cardiotoxicity potential (Guo et al., 2011), this approach is not amenable to drugs or chemicals that lack necessary pharmacokinetic studies (i.e., compounds early in development). Thus, we considered an alternative approach to integrating multiple parameters collected in this study to rank chemicals in the screened library for their overall cardiotoxicity and cytotoxicity hazard (Figure 6). Each compound's BMC values for the effects on beat rate, peak shape (spacing, amplitude, rise, decay and width), and cell viability were analyzed and visualized using the ToxPi approach (Reif et al., 2013), which generates transparent graphical rankings to facilitate decision making (see Supplemental Tables 5 and 6 for ToxPi input data files). The analysis of the data for both 30 min (Figure 6A) and 24 hrs (Figure 6B) time points showed that non-toxic compounds were ranked high for safety, while most cardiotoxic compounds were ranked low. Significant correlation of ToxPi values was observed between $30 \mathrm{~min}$ and $24 \mathrm{hr}$ time points $\left(r_{\text {Pearson }}=0.65, r_{\text {Spearman }}=0.62\right)$.

\section{Discussion}

This work was aimed at addressing several major barriers that preclude wide use of human iPSC-derived cardiomyocytes as an in vitro model for cardiotoxicity screening, such as (i) throughput, (ii) data integration across multiple parameters, and (iii) quantitative assessment of the concentration-response. Despite some limitations of iPSC-derived cardiomyocytes (Scott et al., 2013) (e.g., neonatal phenotype, mixed culture of various cardiomyocyte subtypes, un-physiological two-dimensional alignment of cells in culture, etc.), their use represents a future of cardiotoxicity testing. A virtually unlimited supply of functionally competent cells (i.e., spontaneously and synchronously contracting), and a promise to create cells from multiple individuals, including patients with cardiovascular diseases (Liang et al., 2013), make this model system invaluable not only for biomedical research, but also for safety screening of drugs and environmental chemicals.

First advantage of our model system is throughput. The throughput of the technologies that have been reported to date (Qian and Guo, 2010; Guo et al., 2011; Ma et al., 2011; Rana et 
al., 2012; Liang et al., 2013; Pointon et al., 2013) is limited to few or as many as several dozen compounds, and it is not yet clear how the results of the in vitro assays can be used to predict in vivo toxicity (Guo et al., 2011), or to determine additional tests that may be needed. In this study, the cardiac beating assay (Sirenko et al., 2013) was optimized for a 384-well format and the results presented herein show that it is a very promising and robust in vitro phenotyping approach for cardiotoxicity screening in high-throughput format.

Second advantage is the availability of multiple parameters that describe various aspects of impairment in cardiomyocyte physiology. Each output (beat rate, peak characteristics and cell viability) generated as a result of multi-parametric data analysis of the beating patterns of human iPSC-derived cardiomyocytes has tremendous value for understanding the type of pathophysiological effect that a chemical may have on the heart. For example, we observed that a number of cardiotoxic compounds known to prolong the QT interval have a profound impact on the shape of the peaks. Mechanism-based evaluation of clinically-relevant phenotypes (e.g., association of a polymorphic ventricular tachycardia, so called torsades de pointes, that can degenerate into ventricular fibrillation and sudden death, with long QT syndrome) with in vitro screening methods is a promising avenue for triaging drug candidates early in development. Specifically, this effect was observed for Class Ia (disopyramide, procainamide, quinidine) and Class III (amiodarone, sotalol) antiarrhythmic drugs. Other drugs with a similar effect included the commonly used psychotropic medications imipramine and desipramine and the tricyclic antidepressants (TCA), and these medications have been associated with cardiac arrhythmias (Alvarez and Pahissa, 2010).

Similarly, this data can distinguish between $\mathrm{Na}^{+}$channel blockers and hERG blockers. $\mathrm{Na}^{+}$ channel blockers cause increased spacing between peaks (narrow peaks) as well as irregularities of beating patterns. To the contrary, atypical antipsychotics known to act as hERG blockers (Alvarez and Pahissa, 2010) have a profile including dramatic increases of peak width and decay time (wide peaks). The similarity of beating profiles among drugs can potentially provide clues about the mechanisms for toxicity. For example, bupivacaine, a $\mathrm{Na}^{+}$blocker, exhibited a profile different from other $\mathrm{Na}^{+}$blockers, including the appearance of wide peaks. Such a phenotype is consistent with $\mathrm{K}^{+}$blocking and, in fact, bupivacaine has been shown to have such an effect in the literature (Arias et al., 2007). As another example, the anti-inflammatory drug dichlophenac had a very similar profile to $\mathrm{Na}^{+}$channel blockers and, in fact, $\mathrm{Na}^{+}$blocking is a known side effect for this compound (Gwanyanya et al., 2012).

It is also important to point out that the multi-parametric toxicity assessment approach offers an opportunity to distinguish between general (e.g., loss of cell viability) and organ-specific effects. While $\mathrm{Ca}^{2+}$ transients will diminish with the loss of cell viability, an effect observed for many compounds at high enough concentration, detailed concentration-response analysis (see below) and the ability to weigh different effects (e.g., through ToxPi visualization) enables the differential analysis. This is supported by our observation that beat rate and several peak shape parameters were found to be good predictors of cardiotoxicity, while cell viability had poor classification accuracy. These conclusions should be interpreted with caution, however, as our experiments did not test repeated, or longer than $24 \mathrm{hrs}$ exposures.

The third advantage of our approach is in its ability to classify chemicals for potential cardiotoxicity based on quantitative concentration response of multiple parameters. Quantitative assessment of the potential hazard is critical for understanding the safety margins of drugs and chemicals, as well as for the informed decisions on the relative ranking of various drug candidates in development. The most common metric reported from in vitro studies with a concentration-response design to describe the relationship between concentration and effect is $\mathrm{EC}_{50}$ (Neubig et al., 2003). While this metric is applicable to 
radiolabeled ligand-receptor interaction, functional in vitro studies may be conducted at limited ranges of concentrations to avoid non-specific cytotoxicity. The limitations of the $\mathrm{EC}_{50}$ approach in the analysis of in vitro screening data have been recently addressed by Sand et al. (Sand et al., 2012). In addition, an accepted approach to estimating the point-ofdeparture from continuous data for the purpose of human health assessments conducted by the US EPA is the benchmark concentration approach where the benchmark response is defined as one standard deviation of the mean of the control group (EPA, 2012). Because high-throughput in vitro studies usually include many vehicle control wells and few experimental replicates, we propose the use of logistic curve modeling to fit the concentration-response relationships that may not reach the maximum effect, or where it is not known what the maximum effect is due to absence of assay-specific positive controls. Indeed, not only were the BMCs derived using this approach highly predictive of cardiotoxicity hazard (as evident from the AUCs of ROC curves), but our analysis of the alternative point-of-departure cutoffs, up to 3 standard deviations of the mean of controls, demonstrated eroding accuracy of classification.

In high-throughput screening, where multiple phenotypes are derived on many chemicals, the challenge exists to not only compare individual chemicals for each phenotype, but also across various parameters. For this purpose, we reason that the BMC provides a solid basis for standardized comparison among compounds within each phenotype. As some of these phenotypes (e.g., beat rate, peak width) are indicative of particular sub-types of in vivo manifestations of cardiotoxicity, the availability of a value (i.e., BMC) based on best practices in risk assessment should facilitate decisions on the potential for the individual chemicals to cause mechanism-specific toxicity. Still, there are many potential decisionmaking scenarios where a more comprehensive metric is needed to provide a global ranking of compounds for additional in vitro screening in mechanism-based assays or in vivo studies. The ultimate impact of pharmacological toxicity is dependent upon the projected therapeutic margin of safety as well as the risk-to-benefit ratio for new drug entities (Williams, 1990). However, most drugs in development lack pharmacokinetic information necessary to derive a numeric score for estimating the margin of safety. This is similar to the need for assessing the relative risks of environmental chemicals based solely on the data from in vitro assays together with other information such as physico-chemical properties of compounds (Rusyn et al., 2012), exposure estimates, etc. This challenge can be addressed by integrating information across different data domains using ToxPi (Reif et al., 2013), which not only creates a relative rank for the tested compounds, but also provides a comprehensive and transparent visualization of the resultant rankings. Additional data, such as information from other assays, estimates of pharmacokinetic parameters, etc. can be easily incorporated so that diverse sets of chemicals (in environmental screening contexts) or drug candidates from the same development pipeline can be compared based on the diverse evidence (e.g., data) and prioritization needs.

It should be noted that our approach and interpretation of the results are not without limitations. There are several overarching challenges in the use of iPSC-derived cardiomyocytes as an in vitro model for cardiotoxicity screening [reviewed by Scott et al. (Scott et al., 2013)]. One of the impediments is the inability to induce differentiation to a fully mature cardiomyocyte. iPSC cells continue to mature slowly in culture and thus longterm cultures may vary from batch to batch.

Additional caveats that are more specific to high-throughput screening are also worth noting. First, cardiotoxicity as an adverse phenotype is a broad term and the commercial library screened in this study represents compounds with different types of cardiovascular toxicity and/or adverse events. In addition, some of the cardiotoxicity events are acute, some are chronic, and some occur as a consequence of toxicity at other organs. Therefore, despite the 
fact that multiple parameters are being collected in the assay detailed herein, the results should be interpreted with caution and additional phenotypic anchors may need to be collected to improve specificity of the prediction and offer detailed mechanistic understanding for each compound of interest. Nonetheless, we posit that our method shows promise as it allows for rapid and high-throughput screening for both general cytotoxicity and specific cardiomyocyte beating parameters in concentration-response fashion and yields informative ranking and prioritization for additional follow-up studies.

Second, the classification results and interpretation of the predictive value of the individual or combined parameters collected in this assay depend on the assignments of chemicals in the screened library to classes performed by the commercial vendor supplying the library. Indeed, alternative assignments could be made for a number of compounds based on the vast available literature. This limitation may be addressed by additional experiments where mechanism-specific and organ-specific libraries are screened. While detailed assay validation was beyond the scope of this work, we note that as our assignment of compounds to classes was not biased, and that our classification approach which includes collection of multiple parameters and derivation of BMCs should apply to future testing of a greater number of, or alternatively-defined chemicals. Likewise, the use of ToxPi for integration of diverse information across data domains can be utilized for alternative context-dependent decisions.

Third, while we posit that a quantitative approach that explores concentration-response findings in this in vitro system is an advantage which enables decisions that go beyond hazard classification, the challenge of "clinical relevance" is a very important consideration. Careful comparisons of the "effective dose" observed in this assay and a known or estimated blood or tissue concentration of the chemical or drug of interest are needed.

Finally, while the assay was scaled up to a 384-well plate format to facilitates screening of up to 150 compounds per plate, additional experiments are necessary to establish ultra-high throughput approaches, such as 1536-well plates, for a greater number of compounds and concentrations.

\section{Supplementary Material}

Refer to Web version on PubMed Central for supplementary material.

\section{Acknowledgments}

This work was supported, in part, by the National Institutes of Health [ES015241 to I.R.]; and the United States Environmental Protection Agency [STAR RD83516601 to I.R.].

\section{List of abbreviations}

$\begin{array}{ll}\text { iPSC } & \text { induced pluripotent stem cell } \\ \text { BMC } & \text { benchmark concentration } \\ \text { DMSO } & \text { dimethyl sulfoxide } \\ \text { AUC } & \text { area under the curve } \\ \text { EC }_{\mathbf{5 0}} & \text { effective concentration at } 50 \% \text { of the maximum response }\end{array}$




\section{References}

Alvarez PA, Pahissa J. QT alterations in psychopharmacology: proven candidates and suspects. Curr Drug Saf. 2010; 5:97-104. [PubMed: 20210726]

Anson BD, Kolaja KL, Kamp TJ. Opportunities for use of human iPS cells in predictive toxicology. Clin Pharmacol Ther. 2011; 89:754-758. [PubMed: 21430658]

Arias C, Guizy M, David M, Marzian S, Gonzalez T, Decher N, Valenzuela C. Kvbeta1.3 reduces the degree of stereoselective bupivacaine block of Kv1.5 channels. Anesthesiology. 2007; 107:641651. [PubMed: 17893461]

Bers DM. Cardiac excitation-contraction coupling. Nature. 2002; 415:198-205. [PubMed: 11805843]

Cerignoli F, Charlot D, Whittaker R, Ingermanson R, Gehalot P, Savchenko A, Gallacher DJ, Towart R, Price JH, McDonough PM, Mercola M. High throughput measurement of $\mathrm{Ca}(2)(+)$ dynamics for drug risk assessment in human stem cell-derived cardiomyocytes by kinetic image cytometry. $\mathrm{J}$ Pharmacol Toxicol Methods. 2012; 66:246-256. [PubMed: 22926323]

EPA, U. Benchmark dose technical guidance. US Environmental Protection Agency; Washington, DC: 2012.

Fawcett T. An introduction to ROC analysis. Patt Recogn Lett. 2006; 27:861-874.

Frid AA, Matthews EJ. Prediction of drug-related cardiac adverse effects in humans--B: use of QSAR programs for early detection of drug-induced cardiac toxicities. Regul Toxicol Pharmacol. 2010; 56:276-289. [PubMed: 19941924]

Guo L, Abrams RM, Babiarz JE, Cohen JD, Kameoka S, Sanders MJ, Chiao E, Kolaja KL. Estimating the risk of drug-induced proarrhythmia using human induced pluripotent stem cell-derived cardiomyocytes. Toxicol Sci. 2011; 123:281-289. [PubMed: 21693436]

Gwanyanya A, Macianskiene R, Mubagwa K. Insights into the effects of diclofenac and other nonsteroidal anti-inflammatory agents on ion channels. J Pharm Pharmacol. 2012; 64:1359-1375. [PubMed: 22943167]

Kola I, Landis J. Can the pharmaceutical industry reduce attrition rates? Nat Rev Drug Discov. 2004; 3:711-715. [PubMed: 15286737]

Laverty H, Benson C, Cartwright E, Cross M, Garland C, Hammond T, Holloway C, McMahon N, Milligan J, Park B, Pirmohamed M, Pollard C, Radford J, Roome N, Sager P, Singh S, Suter T, Suter W, Trafford A, Volders P, Wallis R, Weaver R, York M, Valentin J. How can we improve our understanding of cardiovascular safety liabilities to develop safer medicines? Br J Pharmacol. 2011; 163:675-693. [PubMed: 21306581]

Lawrence CL, Pollard CE, Hammond TG, Valentin JP. In vitro models of proarrhythmia. Br J Pharmacol. 2008; 154:1516-1522. [PubMed: 18516075]

Liang P, Lan F, Lee AS, Gong T, Sanchez-Freire V, Wang Y, Diecke S, Sallam K, Knowles JW, Nguyen PK, Wang PJ, Bers DM, Robbins RC, Wu JC. Drug Screening Using a Library of Human Induced Pluripotent Stem Cell-Derived Cardiomyocytes Reveals Disease Specific Patterns of Cardiotoxicity. Circulation. 2013

Ma J, Guo L, Fiene SJ, Anson BD, Thomson JA, Kamp TJ, Kolaja KL, Swanson BJ, January CT. High purity human-induced pluripotent stem cell-derived cardiomyocytes: electrophysiological properties of action potentials and ionic currents. Am J Physiol Heart Circ Physiol. 2011; 301:H2006-2017. [PubMed: 21890694]

Mercola M, Colas A, Willems E. Induced pluripotent stem cells in cardiovascular drug discovery. Circ Res. 2013; 112:534-548. [PubMed: 23371902]

Neubig RR, Spedding M, Kenakin T, Christopoulos A. International Union of Pharmacology Committee on Receptor N and Drug C; International Union of Pharmacology Committee on Receptor Nomenclature and Drug Classification. XXXVIII. Update on terms and symbols in quantitative pharmacology. Pharmacol Rev. 2003; 55:597-606. [PubMed: 14657418]

Pointon A, Abi-Gerges N, Cross MJ, Sidaway JE. Phenotypic profiling of structural cardiotoxins in vitro reveals dependency on multiple mechanisms of toxicity. Toxicol Sci. 2013; 132:317-326. [PubMed: 23315586]

Puppala D, Collis LP, Sun SZ, Bonato V, Chen X, Anson B, Pletcher M, Fermini B, Engle SJ. Comparative gene expression profiling in human-induced pluripotent stem cell--derived 
cardiocytes and human and cynomolgus heart tissue. Toxicol Sci. 2013; 131:292-301. [PubMed: 22982684]

Qian JY, Guo L. Altered cytosolic Ca2+ dynamics in cultured Guinea pig cardiomyocytes as an in vitro model to identify potential cardiotoxicants. Toxicol In Vitro. 2010; 24:960-972. [PubMed: 20064605]

Rana P, Anson B, Engle S, Will Y. Characterization of human-induced pluripotent stem cell-derived cardiomyocytes: bioenergetics and utilization in safety screening. Toxicol Sci. 2012; 130:117-131. [PubMed: 22843568]

Reif DM, Sypa M, Lock EF, Wright FA, Wilson A, Cathey T, Judson RR, Rusyn I. ToxPi GUI: an interactive visualization tool for transparent integration of data from diverse sources of evidence. Bioinformatics. 2013; 29:402-403. [PubMed: 23202747]

Robin X, Turck N, Hainard A, Tiberti N, Lisacek F, Sanchez JC, Muller M. pROC: an open-source package for $\mathrm{R}$ and $\mathrm{S}+$ to analyze and compare ROC curves. BMC Bioinformatics. 2011; 12:77. [PubMed: 21414208]

Rusyn I, Sedykh A, Low Y, Guyton KZ, Tropsha A. Predictive modeling of chemical hazard by integrating numerical descriptors of chemical structures and short-term toxicity assay data. Toxicol Sci. 2012; 127:1-9. [PubMed: 22387746]

Sand S, Ringblom J, Hakansson H, Oberg M. The point of transition on the dose-effect curve as a reference point in the evaluation of in vitro toxicity data. J Appl Toxicol. 2012; 32:843-849. [PubMed: 22733407]

Scott CW, Peters MF, Dragan YP. Human induced pluripotent stem cells and their use in drug discovery for toxicity testing. Toxicol Lett. 2013; 219:49-58. [PubMed: 23470867]

Sirenko O, Crittenden C, Callamaras N, Hesley J, Chen YW, Funes C, Rusyn I, Anson B, Cromwell EF. Multiparameter in vitro assessment of compound effects on cardiomyocyte physiology using iPSC cells. J Biomol Screen. 2013; 18:39-53. [PubMed: 22972846]

Taboureau O, Jorgensen FS. In silico predictions of hERG channel blockers in drug discovery: from ligand-based and target-based approaches to systems chemical biology. Comb Chem High Throughput Screen. 2011; 14:375-387. [PubMed: 21470179]

Williams PD. The role of pharmacological profiling in safety assessment. Regul Toxicol Pharmacol. 1990; 12:238-252. [PubMed: 2077560] 


\section{HIGHLIGHTS}

- Induced pluripotent stem cell-derived cardiomyocytes are promising in vitro models

- We tested if evaluation of cardiotoxicity is possible in a high-throughput format

- The assay shows benefits of automated data integration across multiple parameters

- Quantitative assessment of concentration-response is possible using iPSC cells

- Multi-parametric screening allows for cardiotoxicity risk assessment 


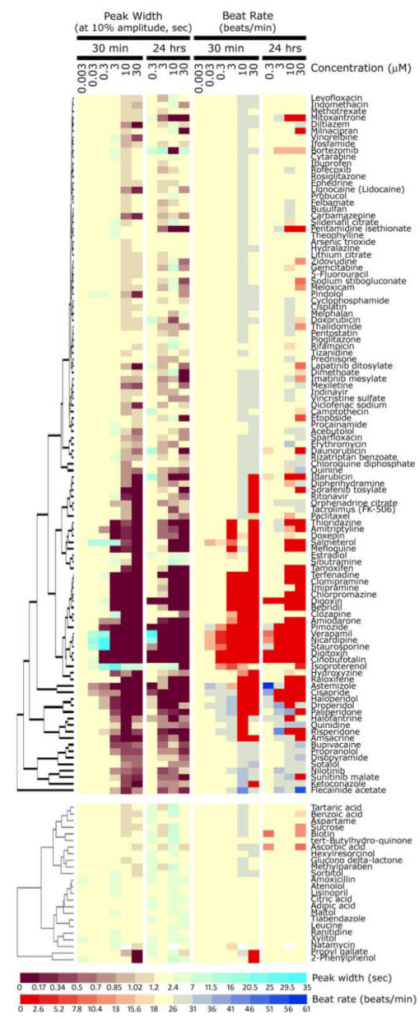

Figure 1.

Concentration-dependent effects of screened compounds on peak width and beat rate of human iPSC-derived cardiomyocytes were visualized using hierarchical clustering and heatmaps. Colors represent changes in each parameter (see color scale bars). Values from duplicate plates were averaged to generate the heatmap. Compounds classified as cardiotoxic in Screen-Well ${ }^{\mathrm{TM}}$ Cardiotoxicity Library (107 chemicals) were clustered separately (top part of the figure) from those classified as non-toxic $(24$, bottom part of the figure). The complete list of compounds (131) used in this study is available as Supplemental Table 1. 

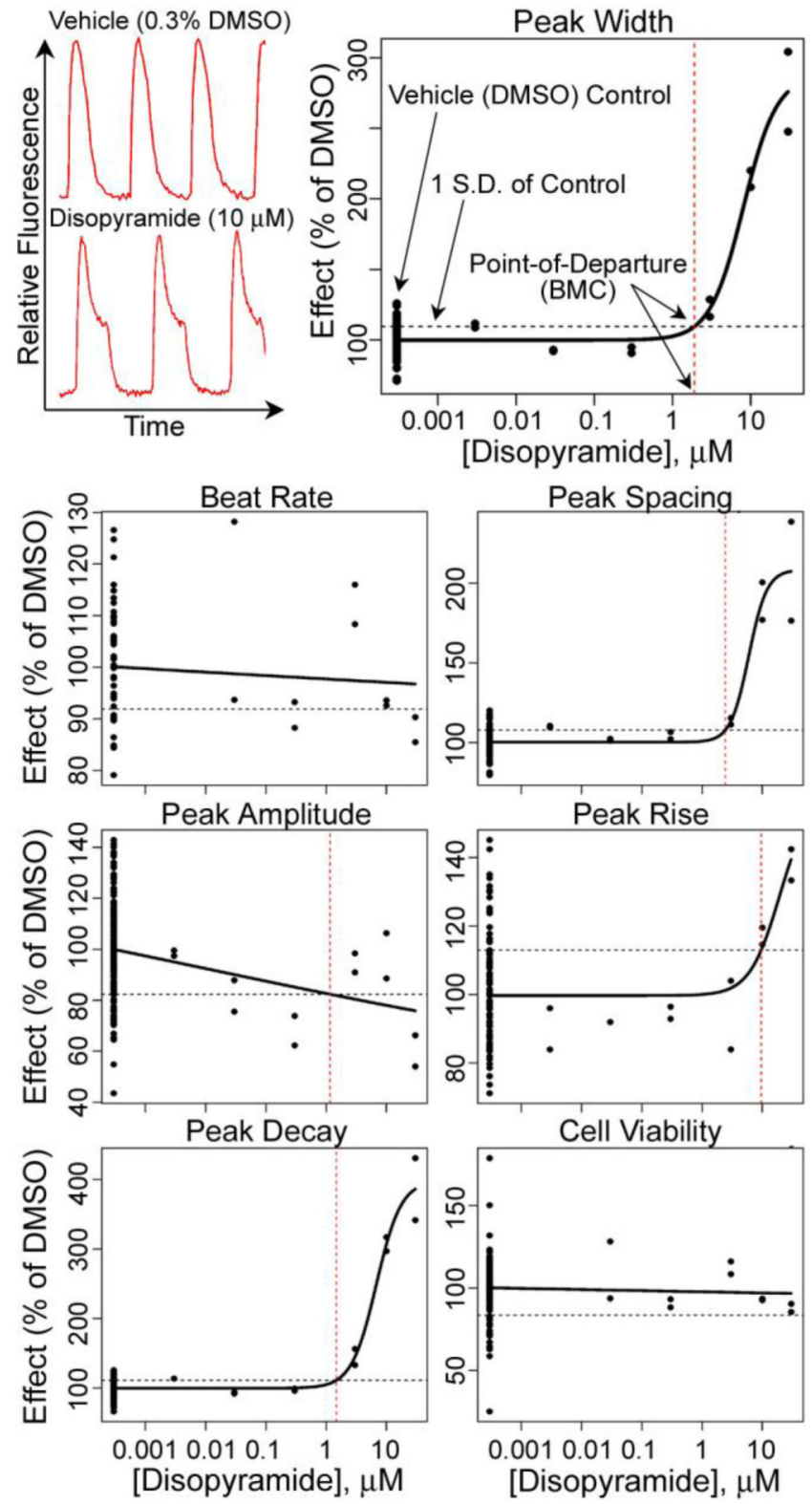

Figure 2.

Quantitative analysis of human iPSC-derived cardiomyocyte beating patterns (top left) was conducted using four-parameter logistic curve fits to the data. Before plotting, individual response values were normalized to plate-specific vehicle (DMSO) control values of each parameter. A benchmark response for the point-of-departure benchmark concentration in the concentration-response curve (vertical dashed lines) was defined as a one standard deviation departure from the mean for vehicle control wells (horizontal dashed lines). 
A 30 min exposure

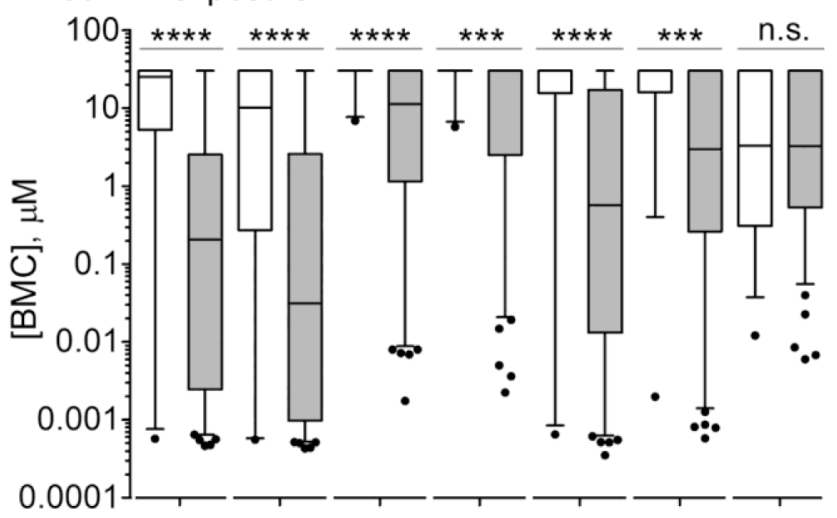

B $24 \mathrm{hr}$ exposure

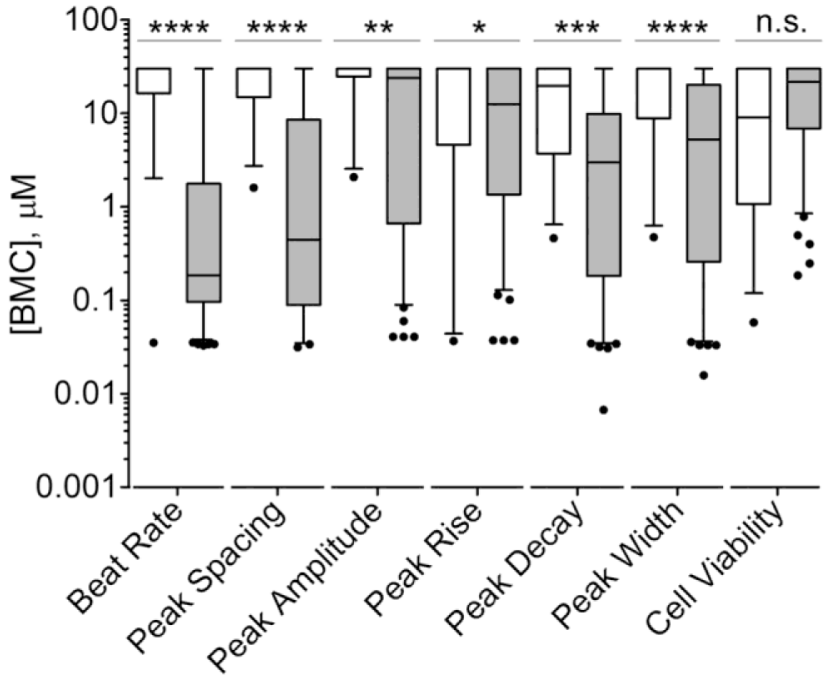

Figure 3.

Distributions of BMC values for non-toxic (white) and cardiotoxic (grey) compounds were plotted using box-and-whiskers plots (line - mean, box - inter-quartile range, whiskers - 5 and 95\%, outliers - dots) for each parameter separately at $30 \mathrm{~min}(\mathbf{A})$ and $24 \mathrm{hr}$ (B) time points. Differences in the means of BMCs between non-toxic and cardiotoxic compounds for each parameter were compared using t-tests and the level of significance is displayed with asterisks $(*, \mathrm{p}<0.05 ; * *, \mathrm{p}<0.01 ; * * *, \mathrm{p}<0.001 ; * * * *, \mathrm{p}<0.0001 ;$ n.s., $\mathrm{p}>0.05)$. 

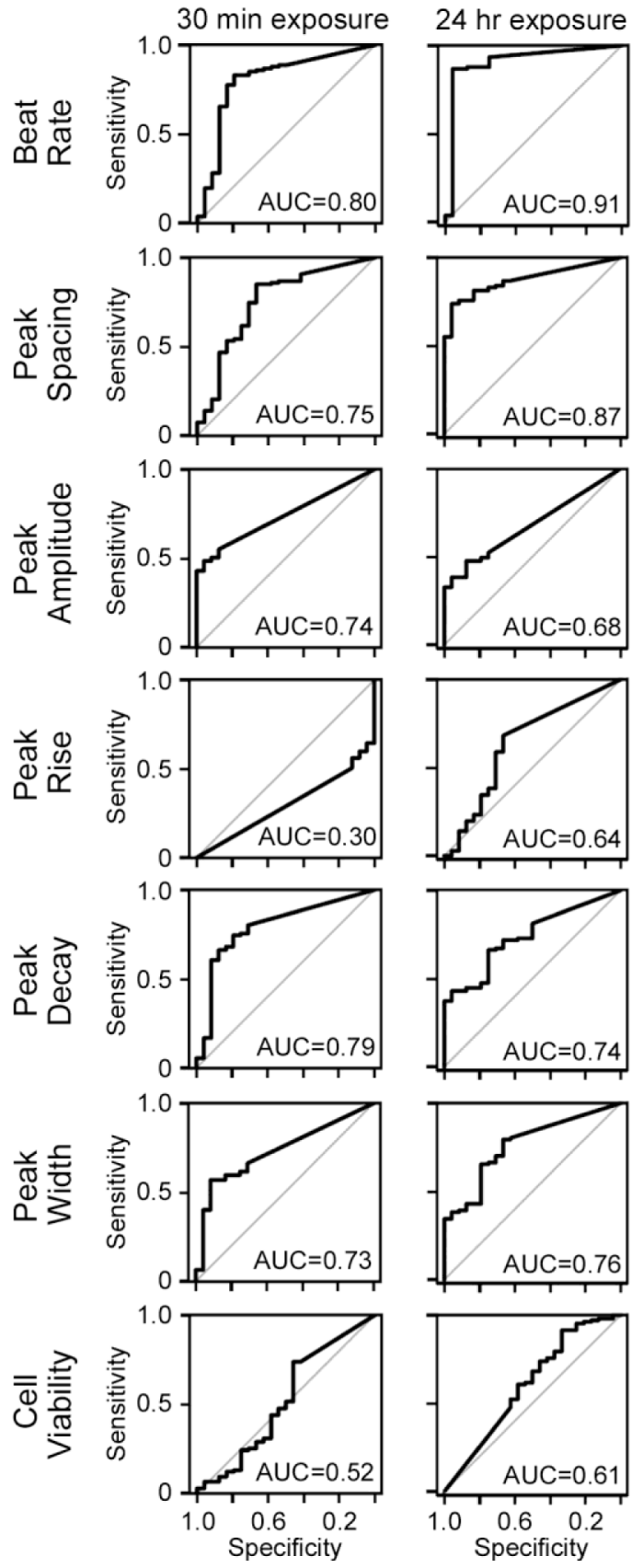

Figure 4.

Receiver Operating Characteristics curves that display the relationship between sensitivity and specificity were obtained from the analysis of the classification accuracy (cardiotoxic vs non-toxic labels of compounds in Screen-Well ${ }^{\mathrm{TM}}$ Cardiotoxicity Library) using BMC values (left - 30 min time point; right $-24 \mathrm{hr}$ time point) for each of the parameters shown. AUC values for each ROC curve is displayed. 

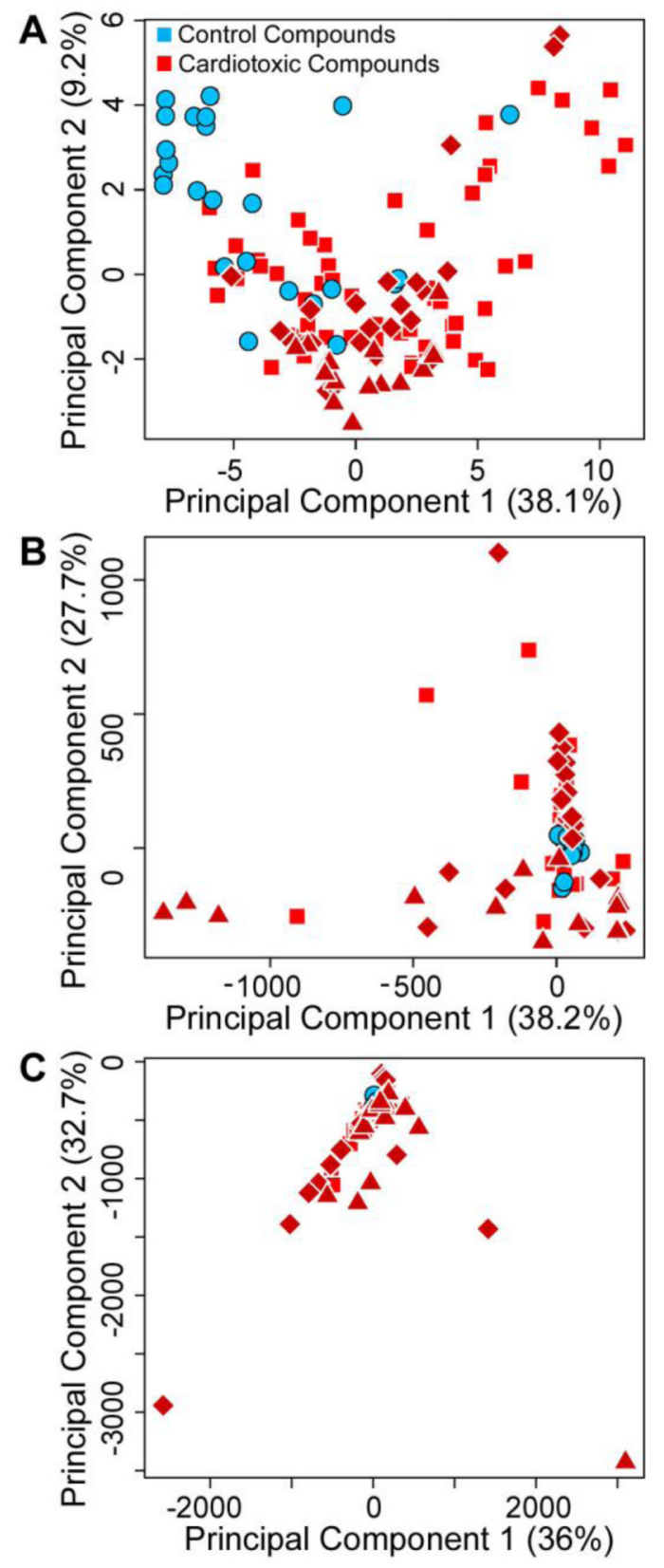

Figure 5.

Principal component analysis (first two principal components shown with percent global variability explained indicated in parentheses) of chemical similarity vs. multi-parametric assessment of cardiotoxicity. A, Chemical diversity of Screen-Well ${ }^{\mathrm{TM}}$ Cardiotoxicity Library drugs using Dragon descriptors. B, Responses at each concentration for beat rate and peak shape (spacing, amplitude, rise, decay and width) for 30 min time point. C, Same as B for 24 hr time point. Non-toxic compounds are shown as blue circles. Cardiotoxic compounds are shown in red (triangles - compounds with known dopamine- and/or histamine-ergic activity, diamonds - compounds with known effects on ion channels, squares - other cardiotoxic compounds). 
A 30 min exposure

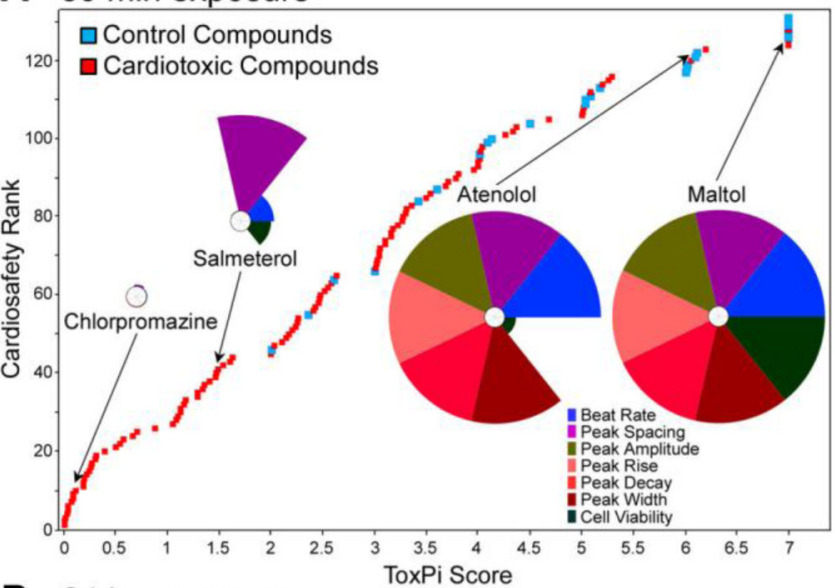

B $24 \mathrm{hr}$ exposure

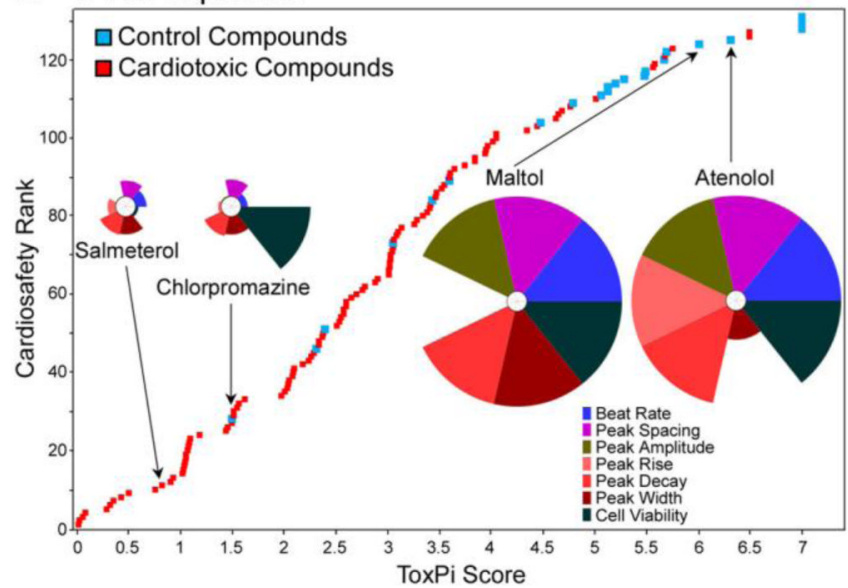

Figure 6.

Relative cardiosafety ranking of Screen-Well ${ }^{\mathrm{TM}}$ Cardiotoxicity Library drugs. ToxPi scores (see Supplemental Tables 5 and 6) were used to create a relative ranking of screened drugs using multiparametric data collected from $30 \mathrm{~min}(\mathbf{A})$ and $24 \mathrm{hr}(\mathbf{B})$ time points. Note that cell viability data for $30 \mathrm{~min}$ time point was collected $24 \mathrm{hrs}$ after addition of chemicals to cells. Blue squares - non-toxic drugs, red squares - cardiotoxic drugs. Representative ToxPi-es for two non-toxic and two cardiotoxic compounds are shown to visualize the relative contribution of each parameter (represented in colors of each slice) to the overall score of a compound. 